els at E9.5; 54 genes, such as those encoding Bmp-1, IGF II and Sox3, were more highly expressed at E13.5; and another 126 genes, such as those encoding Mash 1, FHF-1 and fibulin-2, were expressed at higher levels at E16.5. The identification of these genes with highly stage-specific expression during craniofacial development is potentially important because they are likely to have key roles in the formation of craniofacial tissues. We are currently studying expression patterns of genes from an E8.5-9.5 mouse craniofacial cDNA library to identify novel craniofacial genes by microarray screening approaches.

Lichter, Peter

\section{Disease-specific microarrays for the automated analyses of genetic imbalances by matrix-CGH}

\author{
P. Lichter', M. Nessling'ㄹ, A. Soder', D. Bockㄹ, D. Göttel', S. \\ Solinas-Toldo', S. Stilgenbauer ${ }^{2}$, H. Döhner ${ }^{2} \&$ Stefan Lampel $^{1}$ \\ ${ }^{1}$ Deutsches Krebsforschungszentrum, Im Neuenheimer Feld 280, 69120 \\ Heidelberg, Deutschland \\ ${ }^{2} \mathrm{M}$ edizinische Klinik und Poliklinik V, Heidelberg, Deutschland
}

Comparative genomic hybridization (CGH) has been used for a genome-wide screening of genetic imbalances in tumours. As CGH to chromosomes is technically difficult and yields only limited resolution, the chromosome targets were replaced by arrays of defined DNA fragments, an approach termed matrix-CGH. We have developed a protocol which allows detection of low copy-number gains and losses of genomic segments more than $75 \mathrm{~kb}$ in size, improving the resolution of CGH by two orders of magnitude. High-level amplifications are reliably detected with targets of cosmid size. Detection of high-level amplifications is also achived when tumour test DNA is amplified by a universal PCR procedure due to the limited amount of source material. On automation of the matrix-CGH procedure $^{1}$, we have focused on the development of a disease-specific chip for the diagnosis of recurrently imbalanced regions in chronic lymphocytic leukaemia of Bcell type (B-CLL). The profile of genomic imbalances in B-CLL had been previously assessed in a large series of tumours. DNA fragments from recurrently imbalanced regions were isolated and immobilized on a glass surface. This chip was applied for a screening in a series of B-CLL tumours. Comparison of data obtained by matrix-CGH and interphase cytogenetics revealed a specificity of more than $95 \%$ of matrix-CGH. To further increase the sensitivity and specificity, the chip was modified by including additional targets from the respective regions to obtain redundant informations for the relevant loci. The usefulness of chipbased genomic and expression profiling in B-CLL will be discussed.

\section{Link, Christopher}

\section{Microarray analysis of gene expression in a transgenic Caenorhabditis elegans Alzheimer disease model}

\author{
Chris Link $^{1}$, Amy Fluet ${ }^{1}$, Carolyn J. Johnson ${ }^{1}$, Carrie Van Doren ${ }^{2}$ \\ $\&$ Stuart Kim²
}

${ }^{1}$ Institute for Behavioral Genetics, University of Colorado, Boulder, Colorado 80309, USA

2Department of Developmental Biology, Stanford M edical School, Stanford, California 94305-5427, USA

We have engineered $C$. elegans to express human beta amyloid peptide, which is believed to be central to Alzheimer disease pathology. This expression has clear pathological effects on these transgenic animals. Characterisation of transgenic animals expressing wild-type or variant beta amyloid peptide indicates that these physiological effects are due to a specific, intrinsic toxicity of the beta amyloid peptide, and not due to non-specific results of foreign protein expression. We have therefore employed microarray hybridisation to examine gene expression changes resulting from beta amyloid expression in this model system, in hopes of understanding the cellular and molecular basis of beta amyloid toxicity. Our initial analysis of two independent beta amyloid/control transgenic pairs, using arrays with 11,990 gene-specific probes, has identified approximately 40 genes that appeared to up-regulated twofold or more in both experiments. Encouragingly, prominent among this set are genes encoding small heat shock proteins, which we have previously demonstrated to be strongly up-regulated in response to beta amyloid peptide expression. We have also initiated microarray analysis of transgenic animals containing suppresser mutations that block phenotypes resulting from beta amyloid expression. Further expression comparisons will serve to refine this list of candidate beta amyloid-responsive genes, which will be subsequently explored in mammalian systems.

\section{Liu, Jianjun}

\section{Further analysis of the mechanism of copper trafficking pathways using yeast expression arrays}

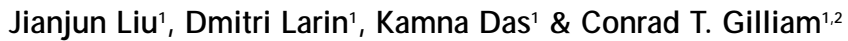 \\ Columbia Genome Center ${ }^{1}$ and Department of Genetics and Development ${ }^{2}$, \\ College of Physicians \& Surgeons, Columbia University, \\ New York, New York 10032, USA
}

Copper is an essential element that serves as cofactor for a number of oxygenprocess enzymes involved in diverse biological processes. In excess, copper ions are highly toxic, thus proper copper trafficking is essential to cell vitality. Mutations in two copper transporting ATPases (ATP7A, ATP7B) lead to disorders of copper starvation (Menkes Disease) and toxicity (Wilson Disease), respectively, in humans. Recent studies show that the molecular components of copper trafficking pathways are highly conserved between yeast and human. In yeast, extracellular $\mathrm{Cu}^{2+}$ is reduced to $\mathrm{Cu}^{+}$by metaloreductase enzymes encoded by fre 1, fre 2, fre 7 and possibly other genes. Next, CTR1 and CTR3 transport $\mathrm{Cu}^{+}$into the cell where cytoplasmic $\mathrm{Cu}^{+}$-chaperones deliver the metal to specific copper-dependent enzymes. LYS7 delivers $\mathrm{Cu}^{+}$to $\mathrm{Cu} / \mathrm{Zn}$-superoxide dismutase, COX17 to cytochrome oxidase and ATX1 to CCC2. CCC2 is the yeast homologue of the Wilson and Menkes disease genes in humans. Ccc2 is located in the trans-Golgi network and transfers $\mathrm{Cu}^{+}$to fet 3 oxidase, which is a homologue of human ceruloplasmin. In yeast, two transcription factors are known to regulate copper metabolism, ACE1 and MAC1. Under conditions of copper starvation, MAC1 is stable and is bound to the copper-responsive promoter elements (CuREs) of fre1, fre2, fre 7, ctr 1 and $c t r 3$. Under conditions of copper excess, MAC1 is rapidly degraded by autoproteolysis, thereby repressing expression of the copper uptake machinery. To further explore the mechanism of copper trafficking, we generated an $S$. cerevisiae strain lacking a functional MAC1 gene. We then compared genomewide expression profiles of the mutant ( $\mathrm{macl}$ ) and isogenic wild-type cells using yeast expression arrays. We will present the results of our expression analyses in light of copper trafficking pathways in yeast and humans. 